\title{
Generación de energía eléctrica mediante el reaprovechamiento de biomasa (cáscara de papa y aguas residuales) por medio de bioceldas. 2016.
}

\author{
Angie Lisette Carrasco Vergara, Elmer Benites Alfaro \\ Universidad Privada César Vallejo, Av. Alfredo Mendiola 6232- Los Olivos, Lima, Perú.
}

Recibido el 15 de noviembre del 2017, aceptado el 20 de diciembre del 2017

DOI: https://doi.org/10.33017/RevECIPeru2017.0010/

\section{Resumen}

El presente trabajo de investigación tuvo como finalidad demostrar que la biomasa (cáscara de papa y agua residual), puede ser reaprovechada en la generación de energía eléctrica mediante bioceldas, para reducir el aumento incontrolable de residuos sólidos, el uso excesivo de energía no renovable y el consumo de energía eléctrica así como de combustibles fósiles. El procedimiento para la investigación se realizó de forma manual, primero se determinó la cantidad de cáscara de papa, luego se extrajo el líquido de glucosa para utilizarlo como sustrato. Después se recolectó el agua residual, y una parte de la muestra se envió a analizar. Posteriormente se procedió a la construcción de la biocelda siguiendo el diseño previamente elaborado, de los cuales la cámara anódica consistió de $357.76 \mathrm{ml}$, la catódica de $265.92 \mathrm{ml}$. Finalmente, se inició el proceso de experimentación con diferentes cantidades de biomasa residual y de esta manera se halló el mejor de los tratamientos. La parte experimental se realizó en un laboratorio de la localidad de Carabayllo, Lima. Para la composición de solución anódica en el Tratamiento 1, se utilizó: a) 35ml del líquido extraído de $100.21 \mathrm{~g}$ de cáscara de papa, b) 250ml de agua residual (obtenida del proceso de lavado de las papas) y c) Material de cultivo biológico (obtenido del agua residual). Para la solución catódica del Tratamiento 1, se utilizó: a) $270 \mathrm{ml}$ de agua destilada $\left(\mathrm{H}_{2} \mathrm{O}\right)$, a la cual se le adicionó Cloruro de Sodio $(\mathrm{NaCl})$ al $2 \%$, para aumentar la conductividad eléctrica. Para los tratamientos 2, 3 y 4 se varío la cantidad de cáscara de papa, siendo 200, 300 y 400 gramos respectivamente. De los resultados obtenidos en la investigación, el tratamiento $\mathrm{N}^{\circ} 4$ evidenció el máximo voltaje, siendo $0.80 \mathrm{~V}$, la intensidad de corriente de $0.1 \mathrm{~A}$ y generando una potencia máxima de $0.080 \mathrm{~W}$, durante los 7 días de experimentación.

Descriptores: Cáscara de papa, agua residual, biocelda y voltaje.

\section{Abstract}

The objective of this research was to demonstrate that biomass (potato peel and wastewater) can be reused in the generation of electric energy by means of biocells to reduce uncontrollable increase of solid waste, excessive use of non-renewable energy and the consumption of electric energy as well as of fossil fuels. The procedure for the investigation was performed manually, first the amount of potato peel was determined, then the liquid was extracted from glucose to be used as a substrate. The residual water was then collected, and a portion of the sample was sent for analysis. Subsequently the construction of the biocelda was carried out following the design previously elaborated, of which the anodic chamber consisted of $357.76 \mathrm{ml}$, the cathodic of $265.92 \mathrm{ml}$. Finally, the experiment was started with different amounts of residual biomass and in this way the best of the treatments was found. The experimental part was carried out in a laboratory in the town of Carabayllo, Lima. For the anodic solution composition in Treatment 1, a) $35 \mathrm{ml}$ of the liquid extracted from $100.21 \mathrm{~g}$ of potato peel, b) $250 \mathrm{ml}$ of waste water (obtained from the potato washing process) and c) Bio culture material (Obtained from wastewater). For the cathodic solution of Treatment 1, a) $270 \mathrm{ml}$ of distilled water $\left(\mathrm{H}_{2} \mathrm{O}\right)$, to which $2 \%$ Sodium Chloride $(\mathrm{NaCl})$ was added, was used to increase the electrical conductivity. For treatments 2, 3 and 4 the amount of potato peel was varied, being 200,300 and 400 grams respectively. From the results obtained in the 
investigation, the treatment No. 4 showed the maximum voltage, being $0.80 \mathrm{~V}$, the current intensity of $0.1 \mathrm{~A}$ and generating a maximum power of $0.080 \mathrm{~W}$, during the 7 days of experimentation.

Keywords: Potato husk, residual water, biocelda.

\section{Introducción}

El crecimiento demográfico de la población mundial es exponencial. Según El Instituto Nacional de Estadística e Informática (INEI), 2015: "En 2011, la población mundial superó los 7.000 millones de personas, y puede que en 2050 alcance los 9.600 millones". El incremento del consumismo en los habitantes, y el uso de materias primas en el mercado es un problema de contaminación ambiental por los desechos que éstos generan durante el proceso de producción o al terminar la vida útil del producto; la fabricación de estos bienes para satisfacer al consumidor se ve en aumento, al igual que el poco interés que muestran las empresas generadoras de éstos residuos al asignarle una adecuada disposición final. [1]

Adicional a ello, los niveles crecientes de industrialización en algunos países, ha aumentado la demanda energética y a la vez ha generado un impacto ambiental por los combustibles fósiles que se utilizan en el proceso. Es por ello que nuestro futuro depende la búsqueda de nuevas fuentes de combustibles alternativos.

La generación de residuos sólidos de manera indiscriminada ocasiona que miles de toneladas al año de residuos orgánicos, lleguen directamente a los vertederos, basureros municipales o incluso cuando no existe una adecuada gestión de residuos sólidos, terminen en los rellenos sanitarios, disminuyendo de esta manera el tiempo de vida útil de estas instalaciones de ingeniería y generando así efectos negativos en el medio ambiente, representando un impacto ambiental considerable.

Si a esta problemática, le sumamos el actual calentamiento global ocasionado por el aumento de los gases de efecto invernadero derivados del uso de hidrocarburos para generar energía, se obtienen diversos impactos ambientales negativos como lo son: La contaminación atmosférica producto de las partículas y gases, contaminación del agua como resultado de la extracción y transporte del petróleo, contaminación del suelo y el calentamiento global, además del agotamiento de recursos no renovables.

El uso incontrolado de los combustibles fósiles ha disparado una crisis energética global, incrementado el interés por obtener fuentes de energía renovables con el mínimo impacto en el medio ambiente. Hasta ahora el compromiso energético de la microbiología ambiental se había dirigido a optimizar la producción de hidrógeno, aprovechar el metano generado en los tratamientos de aguas residuales y en la descomposición de la biomasa residual, o generar biocombustibles como el etanol o el biodiesel. Sin embargo, el reciente descubrimiento de bacterias capaces de convertir energía química en eléctrica sugiere la aparición de una nueva forma de energía verde, cuya explotación supondrá un importante reto biotecnológico en los próximos años.

Además los residuos como producto de las actividades humanas, constituyen un problema creciente, ya que estos desechos se van incrementando de forma directamente proporcional al crecimiento de la población.

De la búsqueda de soluciones a esta problemática, nace la necesidad de aprovechar de mejor manera la biomasa residual (cáscara de papa y aguas residuales), optando por el uso de bioceldas para la generación de energía eléctrica. Utilizar esta energía recicla al carbón, en contraste con los combustibles fósiles.

Un aspecto fundamental en el desarrollo nuevas tecnologías, es que éstos sean sustentables y eficientes en relación al tema medioambiental, por lo que el aprovechamiento de estos residuos se proyecta como una alternativa viable para el uso de energía sostenible.

Baltazar, Edson (2013). Estudió el funcionamiento de las Celdas de Combustible Microbianas (CCM's) y su caracterización haciendo énfasis en los mecanismos de transporte de electrones y los factores que influyen en la operación de las CCM's. Baltazar utilizó diferentes celdas de combustible microbianas de material acrílico-pexiglass con una arquitectura de una sola cámara para la producción de electricidad durante el tratamiento de un agua residual de una unidad habitacional. En las CCM de 8.4 L se obtuvo un voltaje aproximado a circuito abierto de $1.0 \mathrm{~V}$ y en las CCM's de $156 \mathrm{~mL}$ se obtuvo un voltaje a circuito entre 0.5 y $0.7 \mathrm{~V}$. Con una carga de $1 \mathrm{Kohms}$ los voltajes en las celdas fueron entre 30 y $110 \mathrm{mV}$. [2] 
Alzate Gaviria, Liliana; et. al. (2008) Construyeron una celda de combustible microbiana a escala de laboratorio para la generación de electricidad. La celda consistió de dos cámaras separadas por una membrana de intercambio protónico (PEM). Se utilizaron electrodos de papel carbón y un catolito acuoso burbujeado con aire para proveer $\mathrm{O} 2$ disuelto al electrodo. La generación de potencia en la CCM, se debió a la presencia de bacterias como biocatalizadores en la cámara del ánodo. Las bacterias fueron obtenidas de un inoculo mixto anaerobio de tipo entérico, empleando agua residual sintética (ARS) como sustrato. Se determinó la influencia de la temperatura y el $\mathrm{pH}$ sobre el rendimiento de la celda, encontrando que la mayor densidad de potencia fue generada a temperatura mesofílica de $35 \pm 5^{\circ} \mathrm{C}$ y $\mathrm{pH}$ entre 5 y 6 empleando resistencias de 600 y $1000 \Omega$ se obtuvieron como resultados densidades de 640 y $336 \mathrm{~mW} \cdot \mathrm{m}^{-2}$, generando un voltaje de $0.99 \mathrm{~V}$ y $1.05 \mathrm{~V}$, respectivamente. La eficiencia coulómbica obtenida fue de $59,8 \%$. Con ello se concluye que este tipo de sistemas resultan atractivos para la generación de electricidad y a la vez para la degradación de la fracción orgánica. [3]

Sacco, Natalia; Bonetto, María y Corton, Eduardo (2012), realizaron el diseño de una biocelda cuyo compartimiento anódico tuvo un volumen de $(27 \mathrm{ml})$ y fue descartable, el diseñado se realizó utilizando materiales de bajo costo. Escherichia Coli fue la cepa microbiana ensayada como biocatalizador. La metodología desarrollada se basó en la composición del medio mínimo que fue $5,0 \mathrm{~g}$ glucosa, 6,0 $\mathrm{g} \mathrm{Na}_{2} \mathrm{SO}_{4}$, 3,0 $\mathrm{g} \mathrm{KH}_{2} \mathrm{PO}_{4}, 1,0 \mathrm{~g} \mathrm{NH}_{4} \mathrm{Cl}$, $0,5 \mathrm{~g} \mathrm{NaCl}, 120 \mathrm{mg} \mathrm{MgSO}{ }_{4} .7 \mathrm{H}_{2} \mathrm{O}, 10 \mathrm{mg} \mathrm{CaCl}$, disuelto en 1 litro de agua destilada, y $\mathrm{pH}$ ajustado a $7,0 .[4]$

Villareal Rojas, Ana; et.al. (2015) diseñaron una biocelda para evaluar el uso de un consorcio de bacterias sulfato-reductoras (BSR) en la generación de energía. Su metodología consistió en unir dos cámaras en forma de L con una tuerca que sostenía la membrana de intercambio de protones. Se usaron electrodos de grafito de $40 \mathrm{~cm}^{2}$ de área. La cámara anódica contenía medio de cultivo con inóculo de BSR, la cámara catódica contenía electrolito a pH 7. Se midió la producción de energía como la diferencia de potencial entre electrodos y la remoción de sulfato en el anolíto. Los resultados mostraron una generación máxima de potencial de $0.71 \mathrm{~V}$, el cual se mantuvo durante 9 días en ensayos de 30 días; la biocelda modificada mostró un potencial máximo de $0.5 \mathrm{~V}$, en un ensayo de 9 días. El diseño propuesto de la biocelda permitió evaluar la actividad sulfato-reductora y la electroactividad del consorcio. El potencial alcanzado fue similar al reportado por otros autores, permitiendo concluir que el sistema diseñado es adecuado para estudios de optimización de las variables de este proceso. [5]

Una biocelda es un dispositivo que utiliza microorganismos para convertir la energía química presente en un sustrato en energía eléctrica, esto es posible cuando bajo ciertas condiciones algunos microorganismos transfieren los electrones producidos en su actividad metabólica a un electrodo (ánodo) en lugar de a un aceptor natural de electrones (como oxígeno). Este proceso contribuye a degradar la materia orgánica representada como sustrato o combustible. [6]

En busca de nuevas fuentes de combustibles alternativos como es utilizar la biomasa residual en tecnologías nuevas y amigables con el medio ambiente para la generación de energía eléctrica de forma sostenible, se diseñó una biocelda para demostrar que el uso de residuos orgánicos (como la cáscara de papa), pueden ser utilizados como carburantes para alimentar las celdas y de esta manera promover el potencial recuperable y económico de la biomasa, además de contribuir a disminuir los problemas que afronta la sociedad actual: El aumento de residuos sólidos y la crisis energética. Los resultados comparativos de dicho tratamiento permitieron determinar la cantidad de energía generada en cada tratamiento.

\section{Metodología}

\subsection{Recolección y caracterización de los residuos de cáscara de papa}

Se escogió la papa, ya que tiene elevados estándares de calidad para el consumo humano, por lo tanto los productores y consumidores de los mismos generan una alta cantidad de productos de rechazo, así como una generación de residuos (cáscaras). La recolección y caracterización de los residuos de cáscara de papa se realizaron de forma manual y al azahar. Los residuos fueron picados para reducir su volumen, posteriormente se extrajo el líquido de glucosa y fructuosa de las cáscaras de papa, con ayuda de un extractor y con ello, facilitar la degradación en la celda anódica.

La cáscara de papa se considera uno de los mejores conductores de electricidad por tratarse de un vegetal uniformemente sólido, muy rico en agua 
(alrededor de un $80 \%$ de su composición), en almidón y en potasio, lo que le permite "mover" la corriente generada a través de ella mucho mejor.

Para el funcionamiento de la biocelda, se llenaron las celdas según se indica en la tabla $\mathrm{N}^{\circ} 1$, y se tuvo en cuenta que el agua residual contenía materia bacteriológica.

\subsection{Toma de muestra del agua residual}

El agua residual utilizada para la investigación se recuperó de una empresa que realiza la actividad de pelado y picado de papa., debido a que durante el proceso de cortado y lavado del tubérculo, se genera abundante líquido residual. Para ello se conversó con el encargado del lugar y se obtuvo 1.5 litros del efluente, del cual: 1 litro se distribuyó como se indica en la tabla $N^{\circ} 1$.

Como pre-tratamiento, se envió a analizar una muestra de 500ml restante, al laboratorio de Ecología Microbiana y Biotecnología de la Universidad Nacional Agraria La Molina para identificar la cantidad de bacterias anaerobias y presencia de E. Coli en el agua residual. Además, se analizó en campo las muestras de agua residual de $250 \mathrm{ml}$, obteniendo los resultados de la tabla $\mathrm{N}^{\circ} 2$.

\subsection{Construcción del prototipo de biocelda}

Consistió en la construcción de un prototipo de biocelda, que constó de dos cámaras de acrílico (una anódica y otra catódica), en forma rectangular unidas por una membrana de intercambio catiónico. La cámara anódica de $357.36 \mathrm{ml}$ (teórico), contuvo la suspensión de bacterias, el electrolito (sustrato) y el agua residual. La cámara catódica de $265.92 \mathrm{ml}$ (teórico), contenía una solución de $\mathrm{NaCl}$ al $2 \%$ y $\mathrm{pH}$ 7. La distancia que existía entre los electrodos fue de $3 \mathrm{~cm}$. Para la unión de las cámaras, y las reacciones electroquímicas, se utilizó una membrana de intercambio catiónico, que se sostuvo entre los acrílicos de la biocelda gracias a la presión que ejercía la unión de pernos, con las celdas aeróbicas y anaeróbicas.

Para los 4 tratamientos, se utilizó el mismo diseño, variando la cantidad de sustrato (cáscara de papa) en cada una.

\section{A) Selección de los materiales:}

Celdas: El ánodo estuvo compuesto por un marco rectangular de acrílico de $15 \times 10 \mathrm{~cm}$ y $4.4 \mathrm{~cm}$ de ancho. En la superficie se realizaron dos orificios, uno rectangular de $8 \mathrm{~mm} \times 4 \mathrm{~mm}$ de grosor, para la salida del electrodo; y otro circular de $6 \mathrm{~mm}$ de diámetro, para el ingreso y evacuación de la solución de cáscara de papa y agua residual. Este último orificio fue sellado con un perno que cumplió la función de tapón evitando el ingreso de oxígeno a la cámara anódica.

El cátodo: Estuvo compuesto por un marco rectangular de acrílico de $15 \times 20 \mathrm{~cm}$ y $3 \mathrm{~cm}$ de ancho. En la superficie también se realizó un orificio rectangular de las dimensiones anteriormente mencionadas para la salida del electrodo.

Electrodos: Los electrodos de trabajo para la investigación fueron dos placas de grafito de las dimensiones que se observan en la figura $N^{\circ} 15$ Cada uno se utilizó en las cámaras anaerobia y aerobia.

Membrana: Para la unión de las cámaras, se utilizó una membrana de $15 \times 20 \mathrm{~cm}$, la cual fue sumergida en solución de $\mathrm{NaCl}$ al $2 \%$ durante 24 horas previo a su uso.

Electrolito: Para la composición de solución anódica en el Tratamiento 1, se utilizó: a) 30ml del líquido extraído de $100.21 \mathrm{~g}$ de cáscara de papa, b) $250 \mathrm{ml}$ de agua residual (obtenida del proceso de lavado de las papas) y c) Material de cultivo biológico (obtenido del agua residual). Para la solución catódica del Tratamiento 1 , se utilizó: a) $270 \mathrm{ml}$ de agua destilada ( $\mathrm{H} 2 \mathrm{O})$ con conductividad inicial de $0,055 \mu \mathrm{S} / \mathrm{cm}-1$, a la cual se le adicionó Cloruro de Sodio $(\mathrm{NaCl})$ al $2 \%$,

Sustrato: Como combustible a partir del cual se generó energía, se utilizó la glucosa contenida en la cáscara de papa y las aguas residuales del lavado de la misma, con el fin de utilizar la biomasa presente en aguas residuales de diverso tipo.

\section{B) Funcionamiento de la biocelda}

La reacción electroquímica que sucede en el interior de la biocelda se lleva a cabo de la siguiente forma: El sustrato y agua residual es oxidado anaeróbicamente por los microrganismos presentes en el ánodo, produciendo $\mathrm{CO}_{2}$, electrones y protones, los cuales toman diferentes caminos migrando hacia el cátodo.

El hidrógeno o protón $\left(\mathrm{H}^{+}\right)$migra hacia el cátodo y lo hará a través del electrolito (agua residual + cáscara de papa) y a través de la membrana de intercambio de protones (MIP), mientras que los electrones del ánodo, lo hacen a través del electrodo (grafito) y por 
medio de un circuito externo. En el cátodo, un oxidante (normalmente $\mathrm{O}_{2}$ presente en el agua) es reducido para formar $\mathrm{H}_{2} \mathrm{O}$.

De este proceso, para conocer la cantidad de voltaje generada en cada repetición, se utilizó un multímetro y el periodo de toma de datos fue de cada hora.

\section{Resultados}

A continuación se detalla los resultados obtenidos con el programa estadístico Minitab. Los resultados se grafican seguidamente:

\section{Resultados de la Cantidad de Energía}

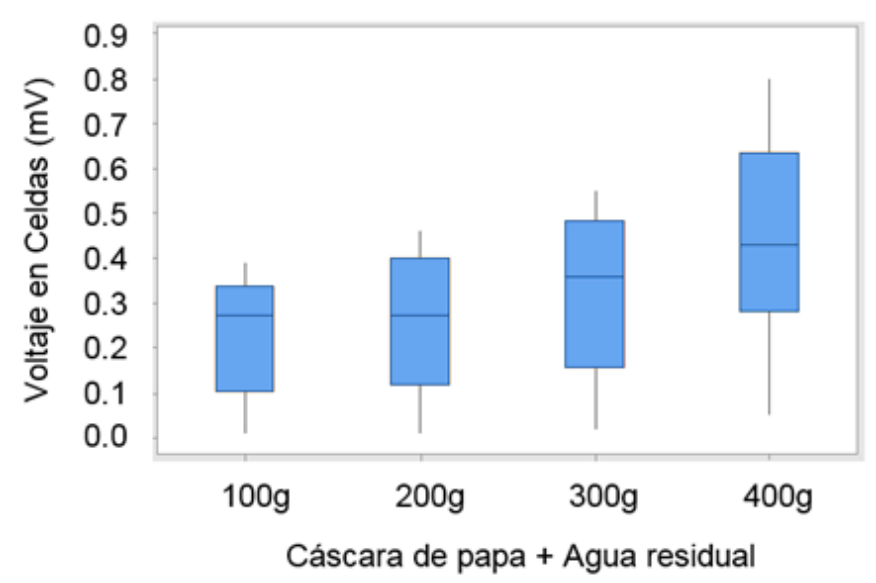

Figura 1: Comportamiento del Voltaje en las bioceldas con respecto a la cantidad de cáscara de papa y agua residual.

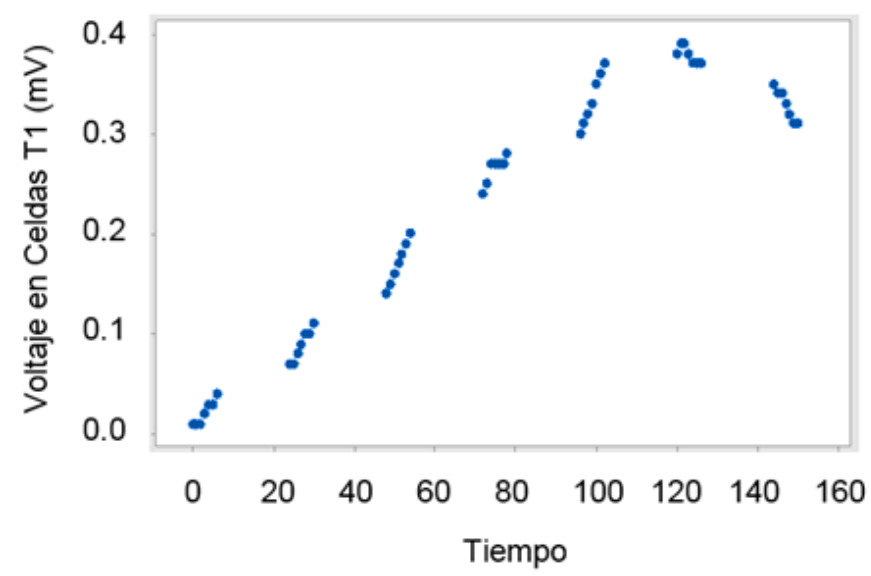

Figura 2: Comportamiento del Voltaje en la biocelda T1 con respecto al tiempo.

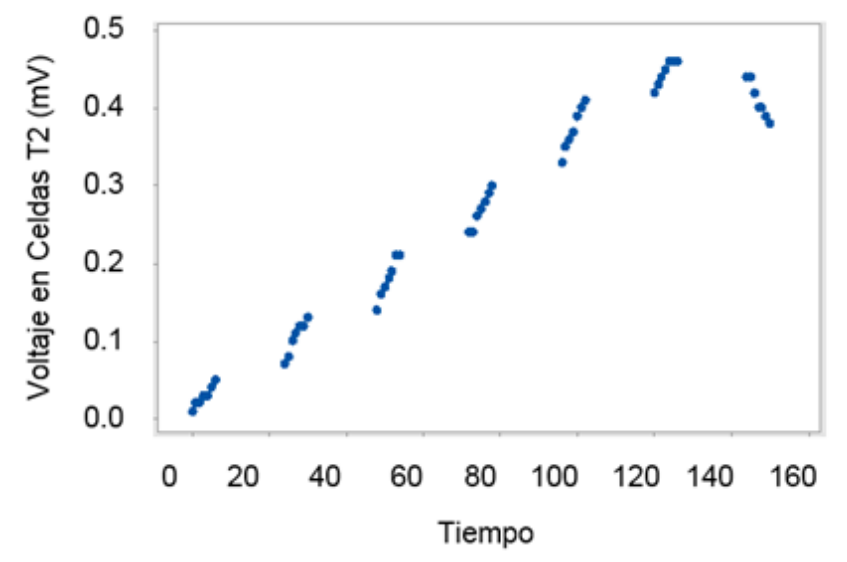

Figura 3: Comportamiento del Voltaje en la biocelda T2 con respecto al tiempo.

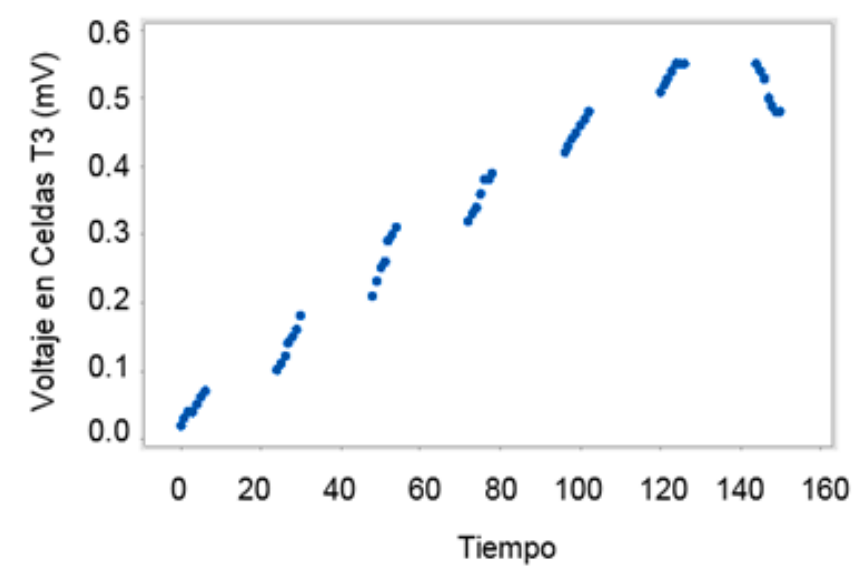

Figura 4: Comportamiento del Voltaje en la biocelda T3 con respecto al tiempo.

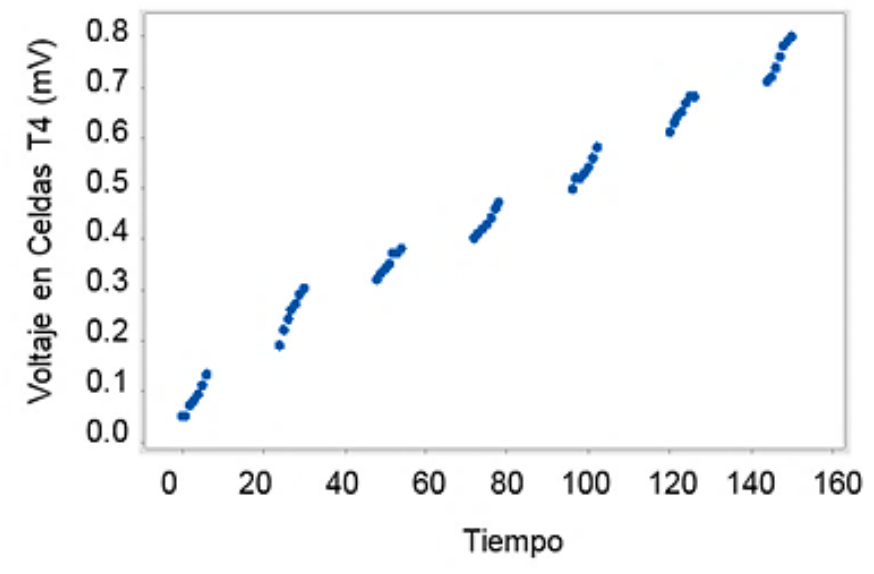

Figura 5: Comportamiento del Voltaje en la biocelda T4 con respecto al tiempo. 


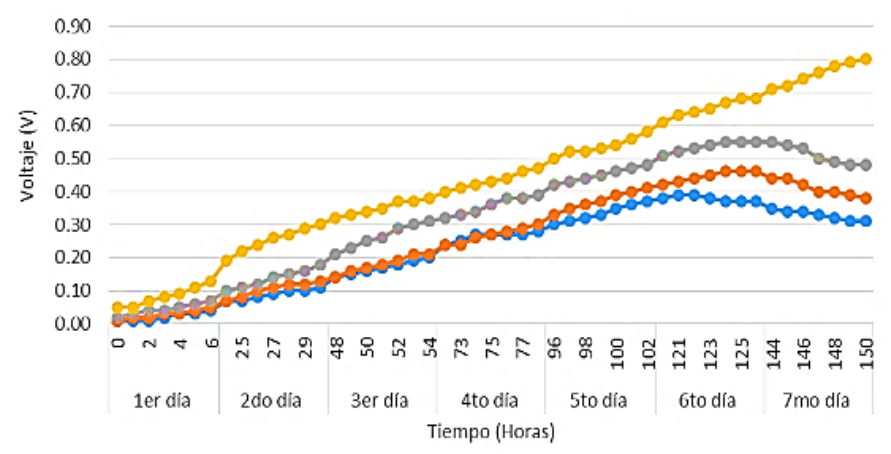

Figura 6: Tratamientos en bioceldas (Voltaje vs Tiempo)

\section{Resultados de la Muestra de Agua Residual}

Tabla 1: Cantidades de biomasa a utilizar en las repeticiones

\begin{tabular}{|c|c|c|}
\hline \multicolumn{3}{|c|}{ Celda Anódica } \\
\hline & Cantidad & Unidades \\
\hline \multicolumn{3}{|l|}{ Tratamiento 1: } \\
\hline Cáscara de papa & 100.21 & $g$ \\
\hline Extracto & 30 & $\mathrm{ml}$ \\
\hline Agua residual & 250 & $\mathrm{ml}$ \\
\hline \multicolumn{3}{|l|}{ Tratamiento 2: } \\
\hline Cáscara de papa & 200.16 & g \\
\hline Extracto & 65 & $\mathrm{ml}$ \\
\hline Agua residual & 250 & $\mathrm{ml}$ \\
\hline \multicolumn{3}{|l|}{ Tratamiento 3: } \\
\hline Cáscara de papa & 300.22 & $g$ \\
\hline Extracto & 90 & $\mathrm{ml}$ \\
\hline Agua residual & 250 & $\mathrm{ml}$ \\
\hline \multicolumn{3}{|l|}{ Tratamiento 4: } \\
\hline Cáscara de papa & 400.01 & $g$ \\
\hline Extracto & 120 & $\mathrm{ml}$ \\
\hline Agua residual & 250 & $\mathrm{ml}$ \\
\hline
\end{tabular}

\section{Celda Catódica}

\section{Para los 4 tratamientos:}

\begin{tabular}{lcc}
$\begin{array}{l}\text { Agua }\left(\mathrm{H}_{2} \mathrm{O}\right) \text { que } \\
\text { ingresa en celda }\end{array}$ & 270 & $\mathrm{ml}$ \\
$\begin{array}{l}\text { Cloruro de Sodio } \\
(\mathrm{NaCl})\end{array}$ & 20 & $\mathrm{~g} / \mathrm{l}$ \\
\hline
\end{tabular}

Tabla 2: Parámetros analizados en campo. Muestra de agua residual

\begin{tabular}{lcc}
\hline Parámetros & Cantid & Unidade \\
\hline Tratamiento 1: & & \\
\hline Conductividad & 856 & $\mu \mathrm{s} / \mathrm{m}$ \\
Temperatura & 22.6 & ${ }^{\circ} \mathrm{C}$ \\
pH & 6.79 & - \\
\hline Tratamiento 2: & & \\
\hline Conductividad & 568 & $\mu \mathrm{s} / \mathrm{m}$ \\
Temperatura & 24.4 & ${ }^{\circ} \mathrm{C}$ \\
pH & 7.58 & - \\
\hline Tratamiento 3: & & \\
\hline Conductividad & 652 & $\mu \mathrm{s} / \mathrm{m}$ \\
Temperatura & 23.1 & ${ }^{\circ} \mathrm{C}$ \\
pH & 7.60 & - \\
\hline Tratamiento 4: & & \\
\hline Conductividad & 573 & $\mu \mathrm{s} / \mathrm{m}$ \\
Temperatura & 23.8 & ${ }^{\circ} \mathrm{C}$ \\
pH & 7.88 & - \\
\hline *Para cada tratamiento se utilizó una \\
muestra de 250ml \\
$\quad$ Fuente: Elaboración propia
\end{tabular}

\section{Interpretación de los resultados}

Con respecto a la energía eléctrica generada a través del reaprovechamiento de biomasa residual por medio de bioceldas, se sostiene que la tecnología de bioceldas, muestran un valor económicamente viable ya que se puede obtener de ella beneficios incuantificables en el aspecto ambiental. Su uso en los sistemas eléctricos aislados puede ser de gran apoyo para proyectos en desarrollo y operación, ya que van de acuerdo con el desarrollo de la nación.

Se sostiene que a mayor dimensión en las celdas, mayor es generación de voltaje. Esto se debe a que existe mayor presencia de electrolito (biomasa) y bacterias que degradan el sustrato para convertirlo en energía. Baltazar, Edson (2013), obtuvo resultados de 0.50 y 0.70 volts en celdas con dimensiones de $156 \mathrm{ml}$, y 1.0 volts en celdas de 8.4 litros, lo que se corrobora con las dimensiones utilizadas en la investigación ( $370 \mathrm{ml}$ para la celda anódica y $270 \mathrm{ml}$ para la celda catódica), siendo el tratamiento $\mathrm{N}^{\circ} 4$, el de mejores resultados por contener la mayor cantidad de biomasa residual.

A. Gaviria (2007), sostiene que el voltaje disminuye gradualmente a medida que el tiempo avanza, esto 
se debe a que la materia orgánica contenida en el electrolito es consumida con el paso de los días por los microrganismos electrogénicos presentes en el medio.

\section{Conclusiones}

En conclusión, el reaprovechamiento de la cáscara de papa y el agua residual si genera energía eléctrica, no obstante, la potencia obtenida para una biocelda es baja en comparación a la cantidad de energía que se necesita para el funcionamiento de sistemas electrónicos de bajo consumo.

De la cantidad de cáscara de papa y agua residual reaprovechada, se concluye que a mayor cantidad de biomasa residual utilizada, mayor es la potencia. Ello se debe a que las bacterias se alimentan de los nutrientes presentes en el electrolito, y cuando estos se agotan, la energía producida desciende, lo que a se evidencia en los 4 tratamientos: Para el Tratamiento $\mathrm{N}^{\circ} 1$ utilizando $100.21 \mathrm{~g}$ (30ml del líquido extraído) y $250 \mathrm{ml}$ de agua residual, el máximo voltaje fue de $0.39 \mathrm{~V}$, la intensidad de corriente de $0.058 \mathrm{~A}$, generando una potencia máxima de $0.019 \mathrm{~W}$, durante los 7 días de experimentación Para la Tratamiento $\mathrm{N}^{\circ} 2$ utilizando $200.16 \mathrm{~g}$ (65ml del líquido extraído) y $250 \mathrm{ml}$ de agua residual, el máximo voltaje fue de $0.46 \mathrm{~V}$, la intensidad de corriente de $0.026 \mathrm{~A}$, generando una potencia máxima de $0.019 \mathrm{~W}$, durante los 7 días de experimentación.

Para la Tratamiento $\mathrm{N}^{\circ} 3$ utilizando $300.22 \mathrm{~g}(90 \mathrm{ml}$ del líquido extraído) y $250 \mathrm{ml}$ de agua residual, el máximo voltaje fue de $0.55 \mathrm{~V}$, la intensidad de corriente de $0.069 \mathrm{~A}$, generando una potencia máxima de $0.038 \mathrm{~W}$, durante los 7 días de experimentación. Para la Tratamiento $\mathrm{N}^{\circ} 4$ utilizando $400.01 \mathrm{~g}$ (120ml del líquido extraído) y $250 \mathrm{ml}$ de agua residual, el máximo voltaje fue de $0.80 \mathrm{~V}$, la intensidad de corriente de $0.1 \mathrm{~A}$, generando una potencia máxima de $0.080 \mathrm{~W}$, durante los 7 días de experimentación.

Finalmente, se concluye que la aplicación primaria de bioceldas es en la producción de bioenergía sostenible, la misma que puede utilizar desechos orgánicos para la generación de electricidad. Este proyecto de ingeniería puede ser usado en lugres que no cuentan con generación de energía convencional.

\section{Agradecimientos}

Al Ing. Benites Alfaro, quién me apoyó durante el desarrollo de la exploración.

Al Ing. Gustavo Román, quién me brindó conocimientos, orientó y motivó a seguir adelante con la investigación.

\section{Referencias}

[1] INEI, Perú: Anuario de Estadísticas Ambientales, (Lima, 2015) 594 p.p.

[2] E. E. Baltazar, Generación de energía eléctrica a partir del tratamiento de aguas residuales por medio de bioceldas. Instituto Mexicano de Tecnología del Agua, 2013.

[3] A. Gaviria, L. Sebastian and P. Hernández, Comparison of two anaerobic systems for hydrogen production from the organic fraction of municipal solid waste and synthetic wastewater, International Journal of Hydrogen Energy, 32 (2007) 3141-3146.

[4] N. Sacco, M. Bonetto y E. Corton, Generación de electricidad en una celda de combustible microbiana mediada utilizando micro-ánodos descartables. Universidad de Buenos Aires, 2012.

[5] A. Villareal, M. Velásquez, C. Estrada, S. Revah, B. Cercado. Evaluación de un consorcio de bacterias sulfatoreductoras en un nuevo diseño de celda microbiana de combustible. Instituto de Ecología, Universidad del Mar, México, 2015.

[6] D. Pant, G. Van, L. Diels y K. Vanbroekhoven. A review of the substrates used cells (MFCs) for sustainable energy production, Bioresource Technology 101 (2010) 1533-1543.

Emails: angiecarrascov92@gmail.com; ebenitesa@ucv.edu.pe 